\title{
NESTS OF SUBSPACES IN BANACH SPACE AND THEIR ORDER TYPES
}

\author{
ALVARO ARIAS AND JEFF FARMER
}

\begin{abstract}
This paper addresses some questions which arise naturally in the theory of nests of subspaces in Banach space. The order topology on the index set of a nest is discussed, as well as the method of spatial indexing by a vector; sufficient geometric conditions for the existence of such a vector are found. It is then shown that a continuous nest exists in any Banach space.

Applications and examples follow; in particular, an extension of the Volterra nest in $L^{\infty}[0,1]$ to a continuous one, a continuous nest in a Banach space having no two elements isomorphic to one another, and a characterization of separable $\mathscr{L}_{p}$-spaces in terms of nests.
\end{abstract}

\section{INTRODUCTION}

We begin with some basic definitions and examples; further background may be found in [L1, L2 and ALWW]; in particular, [L2] contains an excellent bibliography of the subject.

A nest $\mathbf{N}$ is a family of closed subspaces of a Banach space $X$ totally ordered by inclusion. $\mathbf{N}$ is complete if it contains (0), $X$ and the join (closed linear span) and meet (intersection) of any subfamily.

We will consider only complete nests. An index set for a given nest is any ordered set which is order isomorphic to the nest. The order properties of a complete nest are characterized by the order topology of its index set, in the following sense: the meet of any collection of subspaces is the subspace indexed by the order infimum of the indices, and similarly for joins and order suprema.

A nest is said to have a gap between two elements if the index set is disconnected between the two indices; a nest is continuous if it contains no gaps (i.e. its index set is order connected). The index set of a complete nest is always order compact; one may ask of any compact ordered set if it is the index of a nest in some particular space.

A subnest $\mathbf{M}$ of a nest $\mathbf{N}$ is said to be order dense if its completion is $\mathbf{N}$, where the completion of $\mathbf{M}$ is the smallest complete nest containing $\mathbf{M}$. The completion is formed by including meets and joins of all subcollections of nest

Received by the editors July 20, 1989 and, in revised form, January 23, 1990. Presented in AMS sectional meeting, Special Session in Triangular Algebras, Muncie, Indiana, November 1989.

1980 Mathematics Subject Classification (1985 Revision). Primary 46B20; Secondary 46B25, 46B30.

This research was funded in part by the NSF (\#NCS 8703815). The research was initiated in a seminar course given by D. Larson. Portions of this paper form part of the Ph.D. Thesis of each author, under the supervision of W. B. Johnson. 
elements; clearly $\mathbf{M}$ is order dense in $\mathbf{N}$ iff the index set of $\mathbf{M}$ is dense in the index set of $\mathbf{N}$.

It can be shown that any nest in a separable space can have at most countably many gaps [ALWW]; and also that any such nest contains a countable order dense subnest. One can conclude from this that the order type (the index set with order topology) of any nest in separable Banach space is order isomorphic to a compact subset of the reals $(\S 4)$. In $\S 4$ we also show that given any such compact set and any separable Banach space, there exists a nest in the space with that order type.

Next we consider some important motivating examples.

Example (the discrete nest). Let $\left(e_{i}\right)_{i=1}^{n}$ be a basis for $H_{n}=n$-dimensional Hilbert space $\left(n \in \mathbf{Z}^{+}\right)$. Define: $N_{k}=\operatorname{span}\left\{e_{1}, \ldots, e_{k}\right\} \quad \forall k \leq n$. Then

$$
\mathbf{N}=\left\{(0), N_{1}, N_{2}, \ldots, N_{n}=H_{n}\right\}
$$

is a complete nest.

Example (the Volterra nest). $X=L_{p}([0,1], \mu)$ where $\mu$ is Lebesgue measure (or any finite nonatomic Borel measure). For $0 \leq t \leq 1$ let $N_{t}=M_{\chi[0, t]} X$ (the set of all $L_{p}$ functions $\mu$-essentially supported on $\left.[0, t]\right)$ and $\mathbf{N}=\left\{N_{t} \mid 0 \leq\right.$ $t \leq 1\}$. It is routine to verify that for $1 \leq p<\infty$ this nest is complete. Of some interest is the fact that if $p=\infty$ the nest is not complete; for if we let $t=\frac{1}{2}$ (say) and $\mathbf{F}=\left\{N_{s} \mid s<\frac{1}{2}\right\}$, then

$$
L=\bigvee\{N \mid N \in \mathbf{F}\}
$$

is not in $\mathbf{N}$. It can be verified that the addition of these sets will complete the $L_{\infty}$ nest. The order type of the Volterra nest for $p<\infty$ is $[0,1]$; for $p=\infty$ it is $[0,1] \times\{0,1\}$ in the lexicographic order.

The Volterra nest in $L_{p}([0,1], \mu)$ for $p<\infty$ is a continuous nest. In $\S 3$ we will show that a continuous nest may also exist in a sequence space (we will eventually build such a nest in any Banach space).

Example (the Cantor nest). Let $H$ be a separable Hilbert space. Let $\left(e_{r}\right)_{r \in Q}$ be an orthonormal basis for $H . \forall t \in \mathbf{R}$ define $N_{t}=\overline{\operatorname{span}}\left\{e_{r} \mid r \in \mathbf{Q}, r<t\right\}$ and for all $t \in \mathbf{Q}$ define $M_{t}=\overline{\operatorname{span}}\left\{e_{r} \mid r \in \mathbf{Q}, r \leq t\right\}$. Then let

$$
\mathbf{N}=\left\{N_{t} \mid t \in \mathbf{R}\right\} \cup\left\{M_{t} \mid t \in \mathbf{Q}\right\} \cup\{(0)\} \cup\{H\} .
$$

It is easy to verify that $\mathbf{N}$ is complete. This nest is order isomorphic to the Cantor ternary set.

We now consider the idea of an indexing vector for a nest.

Let $\mathbf{N}$ be a complete nest in a Banach space $X$. For each $x \in X$, the function $\varphi: \mathbf{N} \rightarrow \mathbf{R}$ by $\varphi(N)=\operatorname{dist}(x, N)$ has the following properties.

(i) it is continuous from the order topology on $\mathbf{N}$ to the usual topology on R.

(ii) its range is compact (in fact, $\operatorname{ran}(\varphi) \subset[0,\|x\|]$ ).

(iii) if the map is injective, it is a homeomorphism onto its range.

We can ask the question: If $\mathbf{N}$ is complete and order separable (i.e. has a countable order dense subnest) does there exist a vector $x \in X$ making the above function injective? We call such a vector an indexing vector. 
The vector $f \equiv 1$ is an indexing vector for the Volterra nest, $p<\infty$. In separable Hilbert space, every nest has an indexing vector; in fact, D. Larson has pointed out to us that unitary equivalence of complete nests in separable Hilbert space can be characterized using these vectors. This result provides a proof of the Kadison-Singer theorem on similarity (see [KS]) that does not use the spectral theorem.

Larson has asked which Banach spaces will always contain an indexing vector for an arbitrary nest; we will begin by giving some sufficient conditions for this to be true.

\section{INDEXING VECTORS FOR NESTS}

The main goal of this section is to give sufficient conditions on a Banach space so that every nest in it will have an indexing vector.

Definition. Let $X$ be a Banach space. We say that a nest $\mathbf{N}$ in $X$ has unique best approximations if for every $N \in \mathbf{N}$ and $x \in X$, there exists a unique $n_{x} \in \mathbf{N}$ such that $\operatorname{dist}(x, N)=\left\|x-n_{x}\right\|$. That is, the distance from a subspace of the nest to an outside vector is attained by a unique element of the subspace. If this is true for every possible nest in $X$ we simply say that $X$ has unique best approximations.

Theorem 2.1. Let $X$ be a separable Banach space. If $\mathbf{N}$ is a complete nest in $X$ with unique best approximations, then there exists an indexing vector for the nest $\mathbf{N}$.

We have as an immediate consequence that any complete nest in a separable Banach space which is reflexive and strictly convex (in particular, if $X$ is uniformly convex) will have an indexing vector; for reflexivity guarantees the existence of a best approximating vector for any subspace, and the strict convexity ensures its uniqueness.

The main fact we will need is as follows:

Lemma 2.2. Let $X$ be a Banach space. If a nest $\mathbf{N}$ in $X$ has unique best approximations, with $M \varsubsetneqq N$ elements of $\mathbf{N}$, and if $\operatorname{dist}(x, M)=\operatorname{dist}(x, N)$ and $y \in N \backslash M$ then

$$
\operatorname{dist}(x+y, M)>\operatorname{dist}(x+y, N) .
$$

Proof. By unique best approximations, there exists a unique $m_{x} \in M$ such that $\operatorname{dist}(x, N)=\operatorname{dist}(x, M)=\left\|x-m_{x}\right\|$. Now note that $\operatorname{dist}(x+y, N)=$ $\operatorname{dist}(x, N)$. But there exists a unique $m_{x+y} \in M$ so that $\operatorname{dist}(x+y, M)=$ $\left\|x+y-m_{x+y}\right\|$. If we had $\operatorname{dist}(x+y, M)=\operatorname{dist}(x+y, N)$, we could write

$$
\operatorname{dist}(x, N)=\left\|x+y-m_{x+y}\right\|,
$$

and since the best approximations are unique, we would have $y-m_{x+y}=-m_{x}$ or $y \in M$, a contradiction.

Proof of Theorem 2.1. We construct the indexing vector as follows. Let $\mathbf{S}$ be a countable order dense nest in $\mathbf{N}$. Order (in any fashion) the countable collection of pairs from $\mathbf{S}$ :

$$
\left\{\left(M_{i}, N_{i}\right)\right\}_{i=1}^{\infty} \quad \text { where } M_{i} \varsubsetneqq N_{i} \subset X
$$


Take $x_{1}$ separating $M_{1}$ and $N_{1}$ by (say) $\varepsilon_{1}>0$ with $\left\|x_{1}\right\|=1$ (by this we mean $\left.\operatorname{dist}\left(x_{1}, M_{1}\right)-\operatorname{dist}\left(x_{1}, N_{1}\right)=\varepsilon_{1}\right)$. If $x_{1}$ already separates $M_{2}$ from $N_{2}$ then choose $x_{2}=0$. Otherwise choose $x_{2}$ in $N_{2} \backslash M_{2}$ with $\left\|x_{2}\right\|<\varepsilon_{1} / 8$. Then let

$$
\varepsilon_{2}=\operatorname{dist}\left(x_{1}+x_{2}, M_{2}\right)-\operatorname{dist}\left(x_{1}+x_{2}, N_{2}\right) .
$$

By the main lemma we know that $\varepsilon_{2}>0$. Continuing in this way, we let $x_{n+1}=0$ if $M_{n+1}$ and $N_{n+1}$ are already separated by $s_{n}=\sum_{i=1}^{n} x_{i}$. If not we choose $x_{n+1}$ in $N_{n+1} \backslash M_{n+1}$ with

$$
\left\|x_{n+1}\right\|<\frac{1}{2^{n+2}} \min \left\{\varepsilon_{i}\right\}_{i=1}^{n}
$$

and let

$$
\varepsilon_{n+1}=\operatorname{dist}\left(s_{n+1}, M_{n+1}\right)-\operatorname{dist}\left(s_{n+1}, N_{n+1}\right) .
$$

Again, by the lemma we have $\varepsilon_{n+1}>0$. Define $x=\sum_{i=1}^{\infty} x_{i}$. The claim is that $x$ indexes the nest. Note that it suffices to show that $x$ indexes the order dense subnest $\mathbf{S}$.

So take $M, N \in \mathbf{S}$ with $M \varsubsetneqq N$. Then $\exists k$ such that $M=M_{k}$ and $N=N_{k}$.

Let

$$
s_{k}=\sum_{1}^{k} x_{i} \text { and } t_{k}=\sum_{k+1}^{\infty} x_{i} .
$$

Then, using the triangle inequality and the estimates on the $\left\|x_{i}\right\|$ 's we get

$$
\begin{aligned}
\operatorname{dist} & \left(x, M_{k}\right)-\operatorname{dist}\left(x, N_{k}\right) \\
& \geq \operatorname{dist}\left(s_{k}, M_{k}\right)-\operatorname{dist}\left(t_{k}, M_{k}\right)-\left[\operatorname{dist}\left(s_{k}, N_{k}\right)+\operatorname{dist}\left(t_{k}, N_{k}\right)\right] \\
& =\operatorname{dist}\left(s_{k}, M_{k}\right)-\operatorname{dist}\left(s_{k}, N_{k}\right)-\operatorname{dist}\left(t_{k}, M_{k}\right)-\operatorname{dist}\left(t_{k}, N_{k}\right) \\
& \geq \varepsilon_{k}-2 \sum_{i=k+1}^{\infty}\left\|x_{i}\right\|>\varepsilon_{k}-2 \sum_{i=k+1}^{\infty} \frac{\varepsilon_{k}}{2^{i+1}} \\
& =\varepsilon_{k}\left(1-\frac{1}{2^{k}}\right)>0 .
\end{aligned}
$$

So $x$ is an indexing vector for the nest $\mathbf{N}$ in $X$.

Using basically the same construction and the triangle inequality we can notice that the order type of a nest can be important in determining the existence of an indexing vector.

Corollary 2.3. If a nest $\mathbf{N}$ in $X$ has the order type of the extended positive integers, then the nest has an indexing vector.

The condition of Theorem 2.1 cannot be dropped. We can construct counterexamples in the classical spaces not satisfying the unique best approximation property: $C[0,1], c_{0}, L_{1}[0,1]$.

Example. Consider the space $C[0,1]$. We define a nest indexed by the extended negative integers and zero as follows. Let $\left\{r_{i}\right\}_{i=1}^{\infty}$ be an ordering of the rationals in $(0,1)$. Define

$$
N_{k}=\left\{f \in C[0,1] \mid f\left(r_{i}\right)=0 \forall 0 \leq i \leq-k\right\} \quad \text { where } k \in \mathbf{Z}^{-} .
$$


Of course $N_{0}=C[0,1]$ and $N_{-\infty}=(0)$. One can check that this nest is complete (and even countable) yet has no nonzero indexing vector; it is a simple consequence of the fact that the tails $\left\{r_{i}\right\}_{i=n+1}^{\infty}$ are dense in any subinterval of $(0,1)$.

We can construct a somewhat similar example in the space $c_{0}(\mathbf{Q})$. Let

$$
N_{t}=\overline{\operatorname{span}}\left\{e_{r_{i}} \mid r_{i}<t\right\}
$$

and then form the completion by taking meets and joins. We then have a complete nest with no indexing vector (since for a given $\varepsilon>0$ a $c_{0}$ vector can only have finitely many indices greater than $\varepsilon$ ).

A simple look at Lemma 2.2 could suggest a possible role for finite cotype in the existence of indexing vectors. The next example in $L_{1}[0,1]$ due to W. B. Johnson says that this is not the case.

Proposition 2.4. Let $X$ be a Banach space. Suppose $l: l_{1} \rightarrow X^{*}$ is an isometric embedding such that (defining $l_{i}=x_{i}$ ) the sequence $\left(x_{i}\right)$ either contains a weak $^{*}$ accumulation point of itself or converges weak ${ }^{*}$ to zero. Then there is a nest in $X$ with no indexing vector.

Proof. Define

$$
N_{1 / k}=\left\{\overline{\operatorname{span}}\left\{x_{i}\right\}_{i=1}^{k}\right\}_{\perp} \quad \forall k=1,2, \ldots,
$$

and then let $\mathbf{N}$ be the completion of the nest $\left\{N_{1 / k}: k \geq 1\right\}$. Now if $\mathbf{N}$ were to have an indexing vector, say $x$, then $\operatorname{dist}\left(x, N_{1 / k}\right)$ would be strictly increasing. But

$$
\begin{aligned}
\operatorname{dist}\left(x, N_{1 / k}\right) & =\sup \left\{\left\langle x, x^{*}\right\rangle \mid x^{*} \in N_{1 / k}^{\perp}\left\|x^{*}\right\|=1\right\} \\
& =\sup \left\{\left\langle x, x^{*}\right\rangle \mid x^{*} \in \overline{\operatorname{span}}\left\{x_{i}\right\}_{i=1}^{k}\left\|x^{*}\right\|=1\right\} \\
& =\sup \left\{\left\langle x, x^{*}\right\rangle \mid x^{*} \in\left\{x_{i}\right\}_{i=1}^{k}\right\}
\end{aligned}
$$

(since $\left[x_{i}\right]_{i=1}^{\infty}$ is isometric to $l_{1}$ one may consider only the extreme points) which cannot be strictly increasing under the conditions of the hypothesis.

If we consider the fact that the span of the Rademacher functions in $L^{\infty}[0,1]$ is isometric to $l_{1}$ we may apply the above proposition to obtain a nest in $L^{1}[0,1]$ with no indexing vector. It is interesting to note that the gaps in this nest are infinite dimensional; by a result proved later (Corollary 4.3) this means that it is contained in a continuous nest. We thus obtain a continuous nest in $L^{1}[0,1]$ with no indexing vector.

We conclude this section with some remarks about nests in Banach lattices; for it may be that this is a more appropriate context in which to attempt to generalize some of the theory already developed in Hilbert space.

Consider the Volterra nest in $L_{1}$; note that while $L_{1}$ is neither strictly convex nor reflexive, this nest does have an indexing vector: $f \equiv 1$. This nest satisfies the unique best approximation property; thus the construction above will also apply. This situation can be profitably generalized: by putting some condition on the nests, we can get indexing vectors using different conditions on the Banach space. What we have is this

Proposition 2.5. Let $\mathbf{N}$ be a nest of ideals in an order continuous Banach lattice $X$. If $X$ has the property that for $x, y \in X \quad|x| \wedge|y|=0 \quad x, y \neq 0$ we always have $\|x+y\|>\|x\|$, then $\mathbf{N}$ has unique best approximations. 
As an immediate corollary we get an indexing vector for $\mathbf{N}$.

Proof. Ideals in order continuous Banach lattices are projection bands (this is due to Ando; see [LT, 1.a.11]). Let $M$ be an ideal of $X$ and $P_{M}$ its projection band. Since for every $x \in X, x-P_{M}(x)$ is disjoint from $M$, the hypothesis of the proposition gives that $P_{M}(x)$ is the unique best approximation of $x$.

In particular, if $X$ has a lower p-estimate, i.e., if the $x_{i}$ 's are disjoint then

$$
\left\|\sum_{i=1}^{n} x_{i}\right\| \geq K\left(\sum_{i=1}^{n}\left\|x_{i}\right\|^{p}\right)^{1 / p}
$$

with $K=1$, then we get indexing vectors for nests of projection bands as above.

If we ask whether we can obtain an indexing vector by an equivalent renorming of the space, then we can do more: Any lattice in which $c_{0}$ cannot be finitely lattice represented has a lower $p$-estimate for some $p$ with some constant; it can be renormed to have constant 1 [LT, 1.f.12ff]. In fact, $c_{0}$ itself can be renormed (by $1+\varepsilon$ ) to have the property required in the proposition:

$$
\left\|\left(a_{n}\right)_{n=1}^{\infty}\right\|\|=\|\left(a_{n}\right)_{n=1}^{\infty} \|_{c_{0}}+\varepsilon \sum_{n=1}^{\infty} \frac{\left|a_{n}\right|}{2^{n}} .
$$

This says that we can find an indexing vector for the nest in the second example above under an arbitrarily small renorming. This is in contrast to the example above in $C[0,1]$ (which is not order continuous).

It may thus be that the appropriate interesting nests to study are nests of projection bands in Banach lattices, or perhaps nests with uniformly bounded commuting projections (see, for example [W]). One certainly needs some condition on the nest; for, as we show next, every Banach space has a continuous nest.

\section{A CONTINUOUS NEST IN BANACH SPACES WITH BASES}

The aim of this section is to construct a continuous nest in any Banach space with a basis. We assume that $\left(e_{n}\right)_{i=1}^{\infty}$ is a normalized basis of $X$ with constant 1, i.e.

$$
\left\|\sum_{1}^{n} a_{i} e_{i}\right\| \leq\left\|\sum_{1}^{n+1} a_{i} e_{i}\right\| \quad \forall\left(a_{i}\right)_{1}^{n+1} \text { and } n \in \mathbf{Z}^{+} .
$$

We denote by $e_{i}^{*}$ the biorthogonal functionals, $e_{i}^{*}\left(e_{j}\right)=\delta_{i j}$.

The typical continuous nest in $L^{2}[0,1]$ is the Volterra nest (in fact, in some sense, this is the only continuous nest in $\left.L^{2}[0,1]\right)$. Notice that for this nest, the map

$$
[0,1] \rightarrow L^{2}[0,1]:: t \rightarrow \chi_{[0, t]}
$$

is continuous and that

$$
N_{t}=\overline{\operatorname{span}}\left\{\chi_{[0, s]} \mid 0 \leq s \leq t\right\} .
$$

This is the kind of structure we wish to build in $X$; i.e., we would like to define 
a continuous function $y:[0,1] \rightarrow X$ with the properties:

$$
\begin{aligned}
& y(0)=0, \\
& N_{t}=\overline{\operatorname{span}}\left\{y_{s} \mid 0 \leq s \leq t\right\} \text {, } \\
& t<s \Rightarrow d\left(y_{s}, N_{t}\right)>0, \\
& \mathbf{N}=\left\{N_{t} \mid 0 \leq t \leq 1\right\} \text { is a continuous nest . }
\end{aligned}
$$

We look at what must happen if such a function exists: given $y$ as above, define

$$
g_{n}:[0,1] \rightarrow \mathbf{R}:: t \rightarrow g_{n}(t)=\left\langle y_{t}, e_{n}^{*}\right\rangle \text {. }
$$

It is clear that these $g_{n}$ are continuous and that

$$
y_{t}=\sum_{n=1}^{\infty} g_{n}(t) e_{n}
$$

Since we will want to have $N_{1}=X$, we need the $y_{t}$ to span $X$; we thus need:

$$
x^{*} \in X^{*} \text { and }\left\langle y_{t}, x^{*}\right\rangle=0 \quad \forall t \Rightarrow x^{*}=0
$$

or, in terms of the functions $g_{n}$,

$$
\sum_{n=1}^{\infty}\left\langle e_{n}, x^{*}\right\rangle g_{n}(t)=0 \Rightarrow \forall n \quad\left\langle e_{n}, x^{*}\right\rangle=0 .
$$

The natural candidates for functions having this property are biorthogonal systems and basic sequences in $C[0,1]$. We will take for our functions the classical Schauder basic sequence:

$$
g_{2^{k}+j}(t)= \begin{cases}t-\frac{(j-1)}{2} & \text { for } t \in\left[\frac{2 j-2}{2^{k+1}}, \frac{2 j-1}{2^{k+1}}\right], \\ -t+\frac{j}{2^{k}} & \text { for } t \in\left[\frac{2 j-1}{2^{k+1}}, \frac{j}{2^{k}}\right], \\ 0 & \text { otherwise }\end{cases}
$$

for all $k \in \mathbf{Z}^{+}$and $1 \leq j \leq 2^{k}$ and with $g_{1}(t)=t$.

We then define the vectors which will form the path $y$ : for $t \in[0,1]$

$$
y_{t}=g_{1}(t) e_{1}+\sum_{k=0}^{\infty} \sum_{j=1}^{2^{k}} g_{2^{k}+j}(t) e_{2^{k}+j}
$$

These vectors are well defined since the second sum contains only one nonzero element for each value of $k$ and $\left\|g_{2^{k}+j}\right\|_{\infty} \leq 2^{-(k+1)}$. If $t$ is dyadic, $y_{t}$ is a finite sum.

The continuity of the function $y(t)=y_{t}$ follows easily from the continuity of the functions $g_{n}$ and the fact that

$$
\left\|\sum_{k=N}^{\infty} \sum_{j=1}^{2^{k}} g_{2^{k}+j}\right\|_{\infty} \leq 2^{-N}
$$

We define the nest $\mathbf{N}$ as follows: for $t \in[0,1]$

$$
N_{t}=\overline{\operatorname{span}}\left\{y_{s} \mid 0 \leq s \leq t, s \text { dyadic }\right\} .
$$


Proposition 3.1. $\bigvee_{r<t} N_{r}=N_{t} \quad \forall 0<t \leq 1$.

Proof. If $t$ is not dyadic, the proposition is trivially true. If it is, then it is enough to prove that $y_{t} \in \bigvee_{r<t} N_{r}$. Since $\lim _{r \uparrow t}\left\|y_{t}-y_{r}\right\|=0$ and $\bigvee_{r<t} N_{r}$ is closed, this follows.

Remark. We only used the fact that the function taking $t$ to $y_{t}$ is continuous. The main difficulty will be to establish continuity of the nest in the other direction.

Theorem 3.2. $\bigwedge_{r>t} N_{r}=N_{t} \quad \forall 0 \leq t<1$.

The key to the proof of Theorem 3.2 is to characterize the subspace $N_{t}$ when $t$ is dyadic. In preparation for doing this we will develop some notation and prove some elementary facts about the nest $\mathbf{N}$.

It is convenient to associate with every integer $2^{k}+j$ the dyadic number at which the function $g_{2^{k}+j}$ peaks, $t\left(2^{k}+j\right)=(2 j-1) / 2^{k+1}$ (in the sequel we will always assume when we write this that $\left.1 \leq j \leq 2^{k}\right)$ and vice versa; i.e., if $t=(2 j-1) / 2^{k+1}$ for some $j, k$ then $n(t)=2^{k}+j$. We define the "path" to the vector $y_{t}$ as the set of integers where $g_{n}(t)$ is nonzero:

$$
\text { path } y_{t}=\left\{n \mid g_{n}(t) \neq 0\right\} \text {. }
$$

It is easy to see from the definition that

$$
\text { path } y_{t}=\{1\} \cup\left\{2^{k}+j \mid \frac{j-1}{2^{k}}<t<\frac{j}{2^{k}}, 1 \leq j \leq 2^{k} \text { and } k \geq 0\right\}
$$

and in fact

$$
t \in \operatorname{supp} g_{n} \Leftrightarrow n \in \text { path } y_{t} .
$$

If $t$ is dyadic, i.e. if $t=t\left(2^{q}+p\right)$, then

$$
\text { path } y_{t}=\left\{1,2, n_{1}, \ldots, n_{q}\right\}
$$

where $n_{k}=2^{k}+j_{k}$ and $\left(j_{k}-1\right) / 2^{k}<t<j_{k} / 2^{k}$ with $1 \leq k \leq q$.

Now, let $t$ be any number in $[0,1]$, let $n_{k} \in$ path $y_{t}$, and let $t\left(n_{k}\right)=$ $\left(2 j_{k}-1\right) / 2^{k+1}$ for $k=1,2, \ldots$ Then $\operatorname{supp} g_{n_{k+1}} \subset \operatorname{supp} g_{n_{k}}$. In fact,

$$
\begin{aligned}
& t\left(n_{k}\right)<t \Rightarrow \operatorname{supp} g_{n_{k+1}}=\left(t\left(n_{k}\right), t\left(n_{k}\right)+2^{-(k+1)}\right), \\
& t\left(n_{k}\right)>t \Rightarrow \operatorname{supp} g_{n_{k+1}}=\left(t\left(n_{k}\right)-2^{-(k+1)}, t\left(n_{k}\right)\right) .
\end{aligned}
$$

Notice that $\operatorname{supp} g_{n_{k}}=\left(t\left(n_{k}\right)-2^{-(k+1)}, t\left(n_{k}\right)+2^{-(k+1)}\right)$.

It will be useful to distinguish these two cases; we will say in the first case above that $n_{k}$ is in the "left path" to $y_{t}$, and in the second that it is in the "right path" to $y_{t}$. The left path is an increasing sequence of integers whose associated dyadics form an increasing sequence approaching $t$. Notice that

$$
\text { path } y_{t}=\text { right path } y_{t} \cup \text { left path } y_{t} \cup\{n \mid t(n)=t\}
$$

the last set being empty when $t$ is not dyadic. This decomposition of the path has the following properties. 
Lemma 3.3. Let $t \in[0,1]$ and path $y_{t}=\left\{1,2, n_{1}, \ldots\right\}$. For every $k \geq 1$ (1)-(3) are equivalent:

(1) $n_{k} \in$ left path $y_{t}$.

(2) $t\left(n_{k+1}\right)=t\left(n_{k}\right)+2^{-(k+2)}$.

(3) $t\left(n_{k}\right)<t\left(n_{j}\right) \forall j>k$ (or equivalently $\exists j>k$ ).

We have the analogous equivalences for the right path. We also have the above facts:

(4) path $y_{t\left(n_{k}\right)}=\left\{1,2, n_{1}, \ldots, n_{k}\right\} \quad \forall k \geq 1$.

(5) Whenever $n_{k}$ and $n_{k+r}$ are two "consecutive" elements of left path $y_{t}$ (i.e., if $\left\{n_{k+1}, n_{k+2}, \ldots, n_{k+r-1}\right\} \subset$ right path $\left.y_{t}\right)$ then the left endpoint of supp $g_{n_{j}}$ is $t\left(n_{k}\right)$ for $j=k+1, \ldots, k+r$.

(6) $g_{n_{k}}$ is linear on supp $g_{n_{k+1}}$.

Proof. All but (5) follow quickly from the definitions. For (5) use the formulas for support given above, noting that for elements of right path $y_{t}$, the left endpoint of the support of the Schauder function corresponds with that of the following element.

Let $t=t\left(2^{q}+p\right)$ be a fixed dyadic in $[0,1]$. It follows from (1) that if $\operatorname{supp} g_{n} \subset(t, 1]$ then $\left\langle y_{s}, e_{n}^{*}\right\rangle=0$ for every $s \leq t$. Thus, $\left\langle x, e_{n}^{*}\right\rangle=0$ (we will write $\left.x_{n}=0\right)$. Precisely,

$$
\frac{j-1}{2^{k}} \geq t \Rightarrow x_{2^{k}+j}=0 \quad \forall x \in N_{t} .
$$

Proposition 3.4. For any dyadic $t \in[0,1]$ we have the decomposition

$$
N_{t} \equiv \overline{\operatorname{span}}\left\{e_{2^{k}+j} \mid j / 2^{k} \leq t\right\} \oplus F_{t}
$$

where

$$
F_{t}=\operatorname{span}\left[\left\{y_{t(n)} \mid n \in \text { left path } y_{t}\right\} \cup\left\{y_{t}\right\}\right] .
$$

Remark. If $X=l_{p}$ for $1 \leq p<\infty$ and we take the usual basis, then $N_{t} \approx l_{p}$ for $t$ dyadic. In $\S 5$ we will see that this is true for every $t$ and use it to give a characterization of separable $\mathscr{L}_{p}$-spaces in terms of nests.

Remark. Once we have proved the continuity of the nest $\mathbf{N}$ it will follow that we have a similar formula for $N_{t}$ for nondyadic $t ; F_{t}$ will be defined the same way, but will be infinite-dimensional and not necessarily complemented.

The proposition requires two technical lemmas.

Lemma 3.5. Let $t=t\left(2^{q}+p\right)$ and $P: X \rightarrow \operatorname{span}\left\{e_{n} \mid n \in \operatorname{path} y_{t}\right\}$ be the coordinate projection. If $s \leq t$ then $P y_{s} \in F_{t}$.

Proof. Notice that $P$ is well defined because the path to $y_{t}$ is a finite set of integers. By definition we have that $F_{t}=\operatorname{span}\left\{y_{t_{1}}, y_{t_{2}}, \ldots, y_{t_{m}}\right\}$ for some $0<t_{1}<t_{2}<\cdots<t_{m}=t$. Let $s<t$ and find $i$ such that $t_{i-1} \leq s \leq t_{i}$. From Lemma 3.3(3) we have $n\left(t_{i-1}\right)<n\left(t_{i}\right)$. We claim that for $n \in$ path $y_{t}, g_{n}$ is affine on $\left[t_{i-1}, t_{i}\right]$. For $n>n\left(t_{i}\right), g_{n} \equiv 0$ on $\left[t_{i-1}, t_{i}\right]$ (Lemma 3.3(2) and the definition of $g_{n}$ ). For $n<n\left(t_{i}\right)$ it will suffice (by 3.3(6)) to show that $\left(t_{i-1}, t_{i}\right) \subset \operatorname{supp} g_{n\left(t_{i}\right)}$. But this is clear from 3.3(5). For $n=n\left(t_{i}\right)$ we only need note that there are no elements $m$ of path $y_{t}$ in the interval $\left(t_{i-1}, t_{i}\right)$. Since $g_{n\left(t_{i}\right)}$ "peaks" at $t_{i}$, it is affine in the interval as well. Since $P$ involves only the $g_{n}$ 's in path $y_{t}$ it follows that

$$
P y_{s}=\alpha y_{t_{i-1}}+(1+\alpha) y_{t_{i}}
$$

where $\alpha=\left(t_{i}-s\right) /\left(t_{i}-t_{i-1}\right)$. 
Lemma 3.6. Let $t=t\left(2^{q}+p\right)$. If $j / 2^{k} \leq t$ then $e_{2^{k}+j} \in N_{t}$.

Proof. Let $s=t\left(2^{k}+j\right)$ with $j / 2^{k} \leq t$. Consider path $y_{s}=\left\{1,2, n_{1}, \ldots\right.$, $\left.n_{k}\right\}$. Let $m$ be the first integer such that $\left\{1,2, n_{1}, \ldots, n_{m}\right\} \subset$ path $y_{t}$ and $n_{m+1} \notin$ path $y_{t}$. Since $s<t$ the only possibility is that $n_{m} \in$ left path $y_{t}$ and $n_{m} \in$ right path $y_{s}$ and hence $t\left(n_{i}\right)<t$ for $i=m+1, \ldots, k$. By Lemma 3.3(4) we know that path $y_{t\left(n_{m+1}\right)}=\left\{1,2, n_{1}, \ldots, n_{m+1}\right\}$. Hence for some $c \neq 0$,

$$
y_{t\left(n_{m+1}\right)}=P y_{t\left(n_{m+1}\right)}+c e_{n_{m+1}} \text {. }
$$

Lemma 3.5 says that $P y_{t\left(n_{m+1}\right)} \in F_{t}$; we conclude that $e_{n_{m+1}} \in N_{t}$ because $y_{t\left(n_{m+1}\right)} \in N_{t}$ by definition. If $m+1 \neq k$ we continue, writing for some $c_{1}, c_{2} \neq$ 0 ,

$$
y_{t\left(n_{m+2}\right)}=P y_{t\left(n_{m+2}\right)}+c_{1} e_{n_{m+1}}+c_{2} e_{n_{m+2}} .
$$

This gives $e_{n_{m+2}} \in N_{t}$; going on in this fashion gives the result, and Proposition 3.4 is now immediate.

Two more technical lemmas (to which we refer later as well) are needed.

Lemma 3.7. Let $t=t\left(2^{q}+p\right)$ and $s=t-2^{-(q+2)}$ (i.e., $s$ corresponds to the Schauder function on the next lower level whose support shares a left endpoint with $\left.g_{2^{q}+p}\right)$. If $x \in N_{t}$ then we can find $w \in N_{s}$ so that

$$
\|x-w\| \leq\left|x_{n(t)}\right|+\left\|\sum_{n=2^{q+1}+1}^{\infty} x_{n} e_{n}\right\| .
$$

Proof. By Proposition 3.4 we can write

$$
x=\sum_{j / 2^{k} \leq t} x_{2^{k}+j} e_{2^{k}+j}+P_{t} x
$$

where $P_{t} x \in F_{t}$. Let

$$
w=\sum_{\substack{j / 2^{k} \leq t \\ k \leq q}} x_{2^{k}+j} e_{2^{k}+j}+\left[P_{t} x+x_{n(s)} e_{n(s)}\right] .
$$

Now $w$ clearly satisfies the norm condition. It is easy to compute that if $j / 2^{k} \leq t=(2 p-1) /\left(2^{q}+1\right)$ and $k \leq q$ then $j / 2^{k} \leq s$.

By Lemma 3.8 below $P_{t} x+x_{n(s)} e_{n(s)} \in F_{s}$. So (by Proposition 3.4 again) we see that $w \in N_{s}$.

Lemma 3.8. Let $t=t\left(2^{q}+p\right), s=t-2^{-(q+2)}$ and $x \in \operatorname{span}\left\{e_{n} \mid n \in\right.$ path $\left.y_{s}\right\}$. Then $x \in F_{s}$ if and only if $P_{t} x \in F_{t}$ and $x_{n_{t}}=x_{n_{s}}$.

Proof. Now

$$
x \in F_{s} \subset N_{s} \subset N_{t}=\operatorname{span}\left\{y_{r} \mid r \leq t\right\} .
$$

By Lemma 3.5, $P_{t} x \in F_{t}$. Since

$$
x \in F_{s}=\operatorname{span}\left[\left\{y_{t(n)} \mid n \in \text { left path } y_{s}\right\} \cup\left\{y_{s}\right\}\right]
$$

and for $r \in[0, s]$ we have $g_{n(t)}(r)=g_{n(s)}(r)$ we obtain $x_{n(t)}=x_{n(s)}$. Hence

$$
F_{s} \subset\left\{x \in \operatorname{span}\left\{e_{n} \mid n \in \text { path } y_{s}\right\} \mid P_{t} x \in F_{t} \text { and } x_{n_{t}}=x_{n_{s}}\right\} \equiv E \text {. }
$$


Now notice that the left path $y_{s}=$ left path $y_{t}$ and thus $\operatorname{dim} F_{s}=\operatorname{dim} F_{t}$. Therefore, since $\operatorname{Ker} P_{t} \cap\left[e_{n} \mid n \in\right.$ path $\left.y_{s}\right]=\left[e_{n(s)}\right]$, we also get $\operatorname{dim} E=\operatorname{dim} F_{t}$ and so $E=F_{s}$.

We are finally ready to prove Theorem 3.2 (that the nest is left continuous).

Proof of Theorem 3.2. (Case 1). Suppose $t$ is not dyadic. Then both left path $y_{t}$ and right path $y_{t}$ are infinite sets. Let $x \in \Lambda_{r>t} N_{r}$ and $\delta>0$ be given. Take $N$ so that

$$
\left\|\sum_{i=n}^{\infty} x_{i} e_{i}\right\|<\delta \quad \forall n \geq N
$$

Choose $m>N, m=2^{k}+j$ so that $m \in$ right path $y_{t}$ but $n\left(t(m)-2^{-(k+2)}\right)$ (the next integer in path $y_{t}$ ) is in left path $y_{t}$. Let $s=t(m)-2^{-(k+2)}$ and notice that $s<t<t(m)$ with $t(m)$ and $s$ as in Lemma 3.7. Since $x \in N_{t(m)}$ we can find $w \in N_{s} \subset N_{t}$ satisfying

$$
\|x-w\| \leq\left|x_{m}\right|+\left\|\sum_{i=n(s)}^{\infty} x_{i} e_{i}\right\| \leq 3 \delta
$$

since $n(s)>m>N$. But $\delta$ was arbitrary.

(Case 2). Suppose $t=t\left(2^{q}+p\right)$. Let $x \in \bigwedge_{r>t} N_{r}$. Since

$$
X=\overline{\left[e_{i}\right]_{i=1}^{\infty}}=\overline{\operatorname{span}}\left[\left\{e_{2^{k}+j} \mid \frac{j}{2^{k}} \leq t\right\} \cup\left\{e_{n} \mid n \in \text { path } y_{t}\right\} \cup\left\{e_{2^{k}+j} \mid t \leq \frac{j-1}{2^{k}}\right\}\right]
$$

and

$$
N_{t}=\overline{\operatorname{span}}\left\{e_{2^{k}+j} \mid j / 2^{k} \leq t\right\} \oplus F_{t}
$$

it suffices to show that $x_{2^{k}+j}=0$ for $t \leq(j-1) / 2^{k}$ and that $P_{t} x \in F_{t}$.

Let $s_{i}=t+2^{-(q+1+i)}$ for $i=1,2, \ldots$. Then $s_{i} \downarrow t$. If $t<(j-1) / 2^{k}$ we can find $s_{i}$ such that $t<s_{i}<(j-1) / 2^{k}$; since $x \in N_{s_{i}}$ we have by Proposition 3.4 (specifically comment (3) above it) that $x_{2^{k}+j}=0$. Now if $t=(j-1) / 2^{k}$ then $2^{k}+j=n\left(s_{i}\right)$ for some $i$. Applying Lemma 3.8 repeatedly to the pairs $s_{i}, s_{i+1}$ we see that $x_{n\left(s_{1}\right)}=x_{n\left(s_{2}\right)}=\cdots$. Since $\left(e_{n}\right)$ is a basis, these coordinates must be zero.

That $P_{t} x \in F_{t}$ now follows; for $x_{s_{1}}=0$ implies

$$
P_{t} x=P_{s_{1}} x \in F_{s_{1}}=\operatorname{span}\left[\left\{y_{t(n)} \mid n \in \text { left path } y_{s_{1}}\right\} \cup\left\{y_{s_{1}}\right\}\right] .
$$

But again left path $y_{s_{1}}=$ left path $y_{t}$, so $P_{t} x \in F_{t}$.

\section{CONTINUOUS NESTS AND INDEX SETS IN SEPARABLE BANACH SPACE}

In this section we will use the result of $\S 3$ to construct a continuous nest in any Banach space. We do this by "pasting together" nests formed in quotient spaces; the following lemma is essential.

Lemma 4.1. Let $A$ be a closed subspace of $X, \mathbf{N}$ a continuous nest in $A$. Suppose that $X / A$ is infinite-dimensional, and $\mathbf{M}$ is a continuous nest in a 
closed subspace $B$ of $X / A$. If $\pi$ is the canonical projection onto $X / A$, then $\mathbf{N} \cup \pi^{-1}(\mathbf{M})$ is a continuous nest in $\pi^{-1}(B)$.

Proof. It is clear that we have continuity at every $N \in \mathbf{N}$ such that $N<A$, and continuity from the left at $N=A$. The continuity from the right at $A$ and at every $\pi^{-1}(M)$, follows from the fact that $\pi^{-1}(0)=A$ and that

$$
\pi^{-1}\left(\bigwedge_{\mathbf{M}_{0}} M\right)=\bigwedge_{\mathbf{M}_{0}} \pi^{-1}(M)
$$

for any $\mathbf{M}_{0} \subset \mathbf{M}$. To finish, we show continuity from the left at these places, i.e.,

$$
\pi^{-1}\left(M_{0}\right) \subset \bigvee_{M<M_{0}} \pi^{-1}(M) \quad \forall M_{0} \in \mathbf{M}
$$

(the other inclusion is obvious). Let $x \in \pi^{-1}\left(M_{0}\right)$ and $\varepsilon>0$. Since $\pi x \in$ $M_{0}$ and $\mathbf{M}$ is continuous we can find $M<M_{0}$ and $\pi y \in M$ such that $\|\pi x-\pi y\|_{X / A}<\varepsilon$ which means that there exists $a \in A$ satisfying $\|x-y-a\|<\varepsilon$. Since $y+a \in \pi^{-1}(M)$ we are finished.

Theorem 4.2. There is a continuous nest in every infinite-dimensional Banach space.

Proof. Let $X$ be a Banach space (not necessarily separable). Let $\left(x_{\alpha}\right)_{\alpha \in \Lambda}$ be dense in $B_{X}$. Well order $\Lambda$ and construct a family of closed subspaces in the following way:

We know that any space contains a basic sequence starting with any fixed vector [LT]; choose a basic sequence starting with $x_{1}$,

$$
\left\{x_{1}=e_{1}^{1}, e_{2}^{1}, e_{3}^{1}, \ldots\right\} \text {. }
$$

Construct a continuous nest $\mathbf{N}_{1}$ in $X_{1}=\overline{\operatorname{span}}\left(e_{i}^{1}\right)_{i=1}^{\infty}$ and assume that $X / X_{1}$ is not finite dimensional (if it were, we could extend the basic sequence to a basis for $X$ and finish). Now let

$$
\alpha_{1}=\min \left\{\alpha \mid x_{\alpha} \notin X_{1}\right\} .
$$

Choose a basic sequence $\left(e_{i}^{2}\right)_{i=1}^{\infty}$ of $X / X_{1}$ satisfying $e_{1}^{2}=x_{\alpha_{1}}+X_{1}$ and construct another continuous nest $\mathbf{M}_{2}$ in $\overline{\operatorname{span}}\left(e_{i}^{2}\right)_{i=1}^{\infty}$. By the Lemma we know that $\mathbf{N}_{2}=\mathbf{N}_{1} \cup \pi^{-1}\left(\mathbf{M}_{2}\right)$ is a continuous nest in $X_{2}=\pi^{-1}\left(\overline{\operatorname{span}}\left(e_{i}^{2}\right)_{i=1}^{\infty}\right)$. So we can choose a basic sequence $\left(e_{i}^{3}\right)_{1}^{\infty}$ of $X / X_{2}$ with $e_{1}^{3}=x_{\alpha_{2}}+X_{2}$ where $\alpha_{2}=\min \left\{\alpha \mid x_{\alpha} \notin X_{2}\right\}$ and proceed transfinitely as follows:

If $\alpha$ has an immediate predecessor, let $\mathbf{N}_{\alpha}=\mathbf{N}_{\alpha-1} \cup \pi^{-1}\left(\mathbf{M}_{\alpha}\right)$ as before. If $\alpha$ has no immediate predecessor, let

$$
\mathbf{N}_{\alpha}=\bigcup_{\beta<\alpha} \mathbf{N}_{\beta} \cup\left\{\bigvee_{\beta<\alpha} X_{\beta}\right\}
$$

and of course

$$
X_{\alpha}=\bigvee_{\beta<\alpha} X_{\beta} .
$$

That this process ends is a consequence of the density of the $\left(x_{\alpha}\right)_{\alpha \in \Lambda}$. If the space is separable, we can index this nest by $[0,1]$. 
Now it is easy to see that every nest in a Banach space is contained in some maximal nest. Ringrose [R] proved that maximal nests are either continuous or all of the gaps are one dimensional. Larson has asked whether the Volterra nest in $L_{\infty}$ is contained in a continuous nest. We obtain the positive answer to this question as a corollary to the above theorem.

Corollary 4.3. A nest in a Banach space $X$ is contained in a continuous nest if and only if the gaps in the nest are all infinite dimensional.

Proof. It is clear that if there is a finite-dimensional gap we cannot extend the nest to a continuous one. Going the other way, let $\mathbf{N}=\left\{N_{t} \mid t \in T\right\}$ be a complete nest with infinite-dimensional gaps. We construct a continuous nest in each gap as follows: if $t_{0}$ is the immediate predecessor of $t_{1}$ and $N_{t_{0}} / N_{t_{1}}$ is infinite dimensional, we construct a continuous nest there and lift it as above. Do this for every gap.

If we focus on the index set of a nest it is natural to ask two questions: first, given a nest, what kind of index set can we have (i.e., what is its order type), and second, given an order type (say a compact subset of $\mathbf{R}$ ), is it always possible to construct a nest of that type in various spaces? A result in the direction of the first question is the following.

Proposition 4.4. Let $X$ be a separable Banach space. Let $\mathbf{N}$ be a complete nest in $X$. Then $\mathbf{N}$ is order isomorphic to a compact subset of $[0,1]$.

The proof is elementary; we include it for completeness.

Proof. We will actually define an order isomorphism between the nest and a compact subset of the extended reals. We recall two facts from the introduction. Since $X$ is separable, $\mathbf{N}$ has a countable order dense subnest and at most countably many gaps. If there are only finitely many gaps, it is trivial to define an order isomorphism to $[0,1]$. So let the set of gaps be $G=\left(M_{i}, N_{i}\right)_{i=1}^{\infty}$ (it may be that $M_{i}$ coincides with $N_{j}$ for some values $i, j$ ). We denote by $G$ the union of the elements of pairs from $G$. We choose a countable order dense subnest $\mathbf{M}$ that contains all of the endpoints of the gaps (i.e. $\mathbf{G} \subset \mathbf{M}$ ); it may or may not contain other elements of the nest. We will define the order isomorphism on this subnest and then extend it. We intend first to define a map $\phi: A \subset \mathbf{R} \rightarrow \mathbf{N}$ associating the endpoints of the gaps with endpoints of intervals. Find $\left(a_{i}, b_{i}\right) \subset \mathbf{R}$ such that $M_{i}<M_{j}$ if and only if $a_{i}<b_{i}<a_{j}<b_{j}$ for all $i \in \mathbf{Z}^{+}$. Let

$$
\left(c_{i}, d_{i}\right)=\left(a_{i}+\frac{b_{i}-a_{i}}{4}, b_{i}-\frac{b_{i}-a_{i}}{4}\right)
$$

and let $A=\bigcup\left\{c_{i}, d_{i} \mid i \in \mathbf{Z}^{+}\right\}$. Define

$$
\phi\left(c_{i}\right)=M_{i} \text { and } \phi\left(d_{i}\right)=N_{i} \quad \forall i \in \mathbf{Z}^{+},
$$

$\phi$ is an order homomorphism. The set $A$ contains none of its accumulation points, so we can extend $\phi$ in a well-defined way, $\phi: \bar{A} \rightarrow \mathbf{G}^{*}$ where $\mathbf{G}^{*}$ is the completion of $\mathbf{G}$; when this is done in the obvious way (sups to joins, infs to meets) $\phi$ remains an order homomorphism (it may not be 1-1). By the construction it is easy to see that $\phi$ is at most 2-1 in some places; thus we can pick a nondecreasing function $f: \mathbf{R} \rightarrow \mathbf{R}$ which identifies the pairs 
of points that $\phi$ does and the interval between the pair, but no others. Let $B=f(A)$. Then $B$ is closed and ve can define $g: B \rightarrow \mathbf{G}^{*}$ so that $g$ is an order isomorphism and $f \circ g=\phi$. But now we are almost finished; for if $\mathbf{M} \subset \mathbf{G}^{*}$ then since $\mathbf{G}^{*}$ is complete, $\mathbf{G}^{*}=\mathbf{N}$. However, if $\mathbf{N} \backslash \mathbf{G}^{*}$ is not empty, it contains only elements at which the nest is continuous. These elements must fill in some gaps in $\mathbf{G}^{*}$ since $\mathbf{G}^{*}$ is order isomorphic to a closed subset $B$ of $\mathbf{R}$; it is obvious that we can index these intervals continuously by the appropriate intervals in $\mathbf{R} \backslash B$ in an order-preserving fashion using the countable order-dense subnest. By the previous comments, this completes the proof.

Observe that one can obtain in any Banach space a nest order isomorphic to any compact subset of the unit interval by simply taking a continuous nest in that space and deleting some appropriate elements, we thus have

Corollary 4.5. Let $X$ be a Banach space. For every compact subset $K$ of $[0,1]$ we can find a complete nest $\mathbf{N}$ order isomorphic to $K$.

\section{APPLiCATIONS TO BANACH SPACE THEORY}

This construction of a continuous nest in any space with a basis has some implications for the theory. In this section we will characterize separable $\mathscr{L}_{p^{-}}$ spaces and give examples of the variety of nests that can be constructed in Banach spaces. Our first task will be to prove the following fact about the continuous nest constructed in $\S 3$ (we prove it for every $l_{p}, 1 \leq p<\infty$ ):

Proposition 5.1. Let $\mathbf{N}$ be the continuous nest constructed in $\S 3$ using the canonical basis of $l_{p}$. Then $\sup _{0<t<1} d\left(N_{t}, l_{p}\right) \leq 20$.

The distance here is the Banach-Mazur distance (or condition number):

$$
d(X, Y)=\inf \left\{\|T\|^{-1}\|T\| \mid T: X \rightarrow Y \text { is an isomórphism }\right\} .
$$

In order to prove this proposition we will have to examine the properties of the construction made in $\S 3$ a bit more closely. For $t=t\left(2^{q}+p\right)=(2 p-1) / 2^{q+1}$ we define the next "right" and "left" dyadics to be

$$
r(t)=t+2^{-(q+2)}, \quad l(t)=t-2^{-(q+2)} .
$$

This is just another way of referring to the dyadics associated with integers in a given path; not that if we have $n_{i} \in$ path $y_{t}$ then

$$
\begin{array}{ll}
n_{i} \in \text { left path } y_{t} & \text { iff } t\left(n_{i+1}\right)=r\left(t\left(n_{i}\right)\right), \\
n_{i} \in \text { right path } y_{t} & \text { iff } t\left(n_{i+1}\right)=l\left(t\left(n_{i}\right)\right) .
\end{array}
$$

We will write $r^{2}(t)$ for $r(r(t))$.

Lemma 5.2. Let $t=t\left(2^{q}+p\right)$ and $s=r^{k}(l(t))$. Then for

$$
x \in \operatorname{span}\left\{e_{n} \mid n \in \operatorname{path} y_{s}\right\}, \quad x \in F_{s},
$$

if and only if $P_{t} x \in F_{t}$ and

$$
x_{n_{q}}=x_{n_{q+1}}+2 x_{n_{q+2}}+\cdots+2^{k} x_{n_{q+k+1}} .
$$

Proof. First notice that

$$
\begin{aligned}
& \text { path } y_{t}=\left\{1,2, \ldots, n_{q}\right\}, \quad \text { path } y_{l(t)}=\left\{1,2, \ldots, n_{q}, n_{q+1}\right\}, \\
& \text { path } y_{s}=\left\{1,2, \ldots, n_{q}, n_{q+1}, n_{q+2}, \ldots, n_{q+k+1}\right\} .
\end{aligned}
$$


We also know that $t\left(n_{q+i}\right)<t\left(n_{q}\right)=t$ for $i=1,2, \ldots, k+1$ (Lemma 3.3(3)). The proof will go by induction; first note that the case $k=0$ is Lemma 3.8. We will assume that result true for $s=r^{k}(l(t))$ and prove that it holds for $r(s)=r^{k+1}(l(t))$.

First suppose that

$$
x \in F_{r(s)}=F_{s} \oplus\left[y_{r(s)}\right] .
$$

So we can write $x=z+c y_{r(s)}$ with $z \in F_{s}$ and some $c \in R$. Now $P_{t} x \in F_{t}$ since $P_{t} z \in F_{t}$ (by induction) and $P_{t} y_{r(s)} \in F_{t}$ (by Lemma 3.5). Since $z$ satisfies $(*)$ (again by induction) it of course satisfies

$$
x_{n_{q}}=x_{n_{q+1}}+2 x_{n_{q+2}}+\cdots+2^{k} x_{n_{q+k+1}}+2^{k+1} x_{n_{q+k+2}}
$$

and we can verify directly from the definitions that $y_{r(s)}$ does as well:

$$
\begin{aligned}
\left\langle r_{r(s)}, e_{n_{q}}\right\rangle & =\left\langle y_{s}, e_{n_{q}}\right\rangle+2^{-(q+k+2)}, \\
\left\langle y_{r(s)}, e_{n_{q+i}}\right\rangle & =\left\langle y_{s}, e_{n_{q+i}}\right\rangle-2^{-(q+k+2)} \quad \forall i=1, \ldots, k+1, \\
\left\langle y_{r(s)}, e_{n_{q+k+2}}\right\rangle & =2^{-(q+k+2)} .
\end{aligned}
$$

Thus we have shown that

$$
F_{r(s)} \subset\left\{x \mid x \in \operatorname{span}\left\{e_{n} \mid n \in \text { path } y_{r(s)}\right\}, P_{t} x \in F_{t} \text { and (**) holds }\right\} .
$$

But these subspaces both have the same dimension; namely, $\operatorname{dim} F_{s}+1$. So they must be equal.

From the above lemma it is now obvious that for dyadic $t, F_{t}$ is completely characterized by a set of conditions of the form $(*)$ above. If we take the elements of path $y_{t}$ in order, we see that a new condition is added following each element of right path $y_{t}$ (Lemma 3.8); following each element of the left path an existing condition is modified. The list of conditions will look like this

$$
\begin{aligned}
& x_{1}=x_{2}+2 x_{n_{1}}+\cdots+2^{q} x_{n_{q}} \\
& x_{q}=x_{n_{q+1}}+2 x_{n_{q+2}}+\cdots+2^{k} x_{n_{q+k}}
\end{aligned}
$$

where right path $y_{t}=\left\{1, n_{q}, n_{q+k}, \ldots\right\}$. The number of conditions is given exactly by the number of elements in right path $y_{t}$; this implies that

$$
\operatorname{dim} F_{t}=\text { \# left path } y_{t}+1 \text {. }
$$

The above characterization will allow us to exhibit a nice isomorphism between $F_{t}$ and $l_{p}^{\operatorname{dim} F_{t}}$, proving Proposition 5.1. Suppose the first condition characterizing $F_{t}$ is

$$
x_{1}=x_{2}+2 x_{n_{1}}+\cdots+2^{q} x_{n_{q}} .
$$

We choose the following basis for $F_{t}$ :

$$
\begin{gathered}
v_{1}=e_{1}+2^{-(q-1)} e_{n_{q-1}} \\
v_{2}=e_{2}-2^{-(q-1)} e_{n_{q-1}} \\
v_{3}=e_{n_{1}}-2^{-(q-2)} e_{n_{q-1}} \\
\vdots \\
v_{q}=e_{n_{q-2}}-2^{-1} e_{n_{q-1}} \\
v_{q+1}=-2 e_{n_{q-1}}+e_{n_{q}} .
\end{gathered}
$$


If this is also the last condition defining $F_{t}$ then we finish here. If not, there is a second condition

$$
x_{n_{q}}=x_{n_{q+1}}+2 x_{n_{q+2}}+\cdots+2^{k} x_{n_{q+k}} .
$$

In that case, we continue. We used the element $e_{n_{q-1}}$ rather than $e_{n_{q}}$ above for "cancellation" because $v_{1}, \ldots, v_{q}$ are not affected by the new condition; we redefine $v_{q+1}$ and proceed as follows:

$$
\begin{gathered}
v_{q+1}=-2 e_{n_{q-1}}+e_{n_{q}}+2^{-(k-1)} e_{n_{q+k-1}} \\
v_{q+2}=e_{n_{q+1}}-2^{-(k-1)} e_{n_{q+k-1}} \\
v_{q+3}=e_{n_{q+2}}-2^{-(k-2)} e_{n_{q+k-1}} \\
\quad \vdots \\
v_{q+k-1}=e_{n_{q+k-2}}-2^{-1} e_{n_{q+k-1}} \\
v_{q+k}=-2 e_{n_{q+k-1}}+e_{n_{q+k}} .
\end{gathered}
$$

Again, if this is the last condition we will stop; if not, we will redefine the last basis element (in this case $v_{q+k}$ ) similarly and continue. Observe that the dimensionality conditions above are satisfied. Hence we may proceed in this manner until the set $\left(v_{i}\right)$ spans $F_{t}$. It is clear that these vectors satisfy

$$
\frac{1}{4}\left(\sum_{1}^{j}\left|a_{i}\right|^{p}\right)^{1 / p} \leq\left\|\sum_{1}^{j} a_{i} \frac{v_{i}}{\left\|v_{i}\right\|}\right\|_{p} \leq 5\left(\sum_{1}^{j}\left|a_{i}\right|^{p}\right)^{1 / p} .
$$

Now for nondyadic $t$ we know that $N_{t} \approx l_{p} \oplus F$ with $F$ infinite dimensional (this follows from the proof that the nest is continuous). But we still have a list of conditions characterizing $F_{t}$; now the list is infinite. Since these conditions are of the same form, we can define the sequence $\left(v_{i}\right)$ inductively in exactly the same way. Then $\left\{v_{i}\right\}_{i=1}^{\infty}$ is a basis such that $F \approx l_{p}$ with constant $\leq 20$.

Now 20 is definitely not the best constant here. We do not know if for every $\varepsilon>0$ we can find a nest $\mathbf{N}$ in $l_{p}$ so that $d\left(N, l_{p}\right) \leq 1+\varepsilon$ for every $N \in \mathbf{N}$, but we know that we cannot find one with all of its elements isometric to $l_{p}$.

Proposition 5.3. There is no continuous nest in $l_{p}, 1 \leq p \neq 2<\infty$, having each of its elements isometrically isomorphic to $l_{p}$.

Proof. Assume we can find a continuous nest $N=\left\{N_{t}: 0 \leq t \leq 1\right\}$ such that $N_{t} \equiv l_{p}$ for $0<t \leq 1$. Then for every $t>0$ we can find $\left\{x_{n}^{t}: n \geq 1\right\}$ normalized, disjointly supported such that their closed linear span is equal to $N_{t}$ (by lattice properties of $l_{p}$ ). Notice that even in the case $p=1$ the elements of this nest are weak* closed. For $j \in Z^{+}$let

$$
x(t, j)=\left\{\begin{array}{lll}
x_{n}^{t}, & \text { if }\left\langle e_{j}^{*}, x_{n}^{t}\right\rangle \neq 0 & \text { for some } n \\
0, & \text { if }\left\langle e_{j}^{*}, x_{n}^{t}\right\rangle=0 & \text { for every } n .
\end{array}\right.
$$

It is clear that for every $j \in Z^{+} x(0, j)=0$ and $x(1, j)=e_{j}$; moreover, if $k \neq j$, either $x(t, j)=x(t, k)$ or they are disjointly supported. The main property needed is the fact that the map $t \mapsto\left|\left\langle e_{j}^{*}, x(t, j)\right\rangle\right|$ is nondecreasing and continuous. First observe that $\left|\left\langle e_{j}^{*}, x(t, j)\right\rangle\right|=\left\|e_{j \mid N_{t}}^{*}\right\|$. The continuity of 
the map from below follows directly from the continuity of the nest. To see continuity from above, consider $\left|\left\langle e_{j}^{*}, x(t+1 / n, j)\right\rangle\right|$ for $n=1,2, \ldots$ Take a weak* accumulation point of the set $\{x(t+1 / n, j)\}_{n=1}^{\infty}$; since it is in $N_{t}$ and the map is nondecreasing and left continuous, it must be right continuous. Let $0<r<1$ be such that $0<\left|\left\langle e_{j}^{*}, x(r, j)\right\rangle\right|<1$. Then we can find $k \neq j$ such that $x(r, j)=x(r, k)$. Let $s=\sup \{t: x(t, j)=x(t, k)\}$. Clearly $r \leq s<1$ and $x(s, j)=x(s, k)$. Since for every $\varepsilon>0, x(s+\varepsilon, j) \neq x(s+\varepsilon, k)$ then $\left\langle e_{k}^{*}, x(s+\varepsilon, j)\right\rangle=0$ and $\left|\left\langle e_{k}^{*}, x(s+\varepsilon, j)\right\rangle\right| \geq\left|\left\langle e_{j}^{*}, x(s, j)\right\rangle\right|>0$. As before let $x$ be a weak*-limit of $\{x(s+1 / n, j): n \geq 1\}$. We have that $x \in N_{s}$ (by continuity of the nest) and $\left\langle e_{k}^{*}, x\right\rangle=0$ and $\left\langle e_{j}^{*}, x\right\rangle \neq 0$ contradicting $x(s, j)=x(s, k)$.

We are ready to characterize the separable $\mathscr{L}_{p}$-spaces; first, some definitions:

Definition. Let $X$ be a Banach space. Let $E_{1}, E_{2}$ be subspaces of $X$ of the same finite dimension and $\varepsilon>0$. We say that $E_{2}$ is $\varepsilon$-close to $E_{1}$ if there exists an invertible operator $T$ from $E_{1}$ onto $E_{2}$ with

$$
\|T x-x\| \leq \varepsilon\|x\| \quad \forall x \in E_{1} .
$$

Definition. We say that $X$ is an $\mathscr{L}_{p, \lambda}$-space if given any finite-dimensional $F \subset X$ there exists $E \supset F, \operatorname{dim} E<\infty$ such that $d\left(E, l_{p}^{\operatorname{dim} E}\right) \leq \lambda$. We say

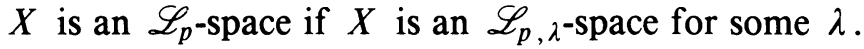

Of course, $L^{p}(\mu)$ is a $\mathscr{L}_{p, \lambda}$-space for any $\lambda>1$.

We are now ready to state the following proposition:

Proposition 5.4. Let $X$ be a separable Banach space. Then $X$ is an $\mathscr{L}_{p}$-space if and only if there exists a continuous nest $\mathbf{N}=\left\{N_{t} \mid 0 \leq t \leq 1\right\}$ in $X$ with $N_{t} \approx l_{p}$ for $0<t<1$ and $\sup _{0<t<1} d\left(N_{t}, l_{p}\right)<\infty$.

The following elementary fact is needed: If $Y, Z$ are Banach spaces with continuous nests $\mathbf{N}$ and $\mathbf{M}$ respectively, indexed by $[0,1]$. Then $\mathbf{N} \oplus \mathbf{M}$ is a continuous nest indexed by $[0,2]$ on $Y \oplus Z ; \mathbf{N} \oplus \mathbf{M}$ is defined by $N_{t}$ if $0 \leq t<1$ and by $Y \oplus M_{t-1}$ if $1 \leq t \leq 2$.

Proof. $(\Rightarrow)$ Let $X$ be a separable $\mathscr{L}_{p}$-space. It follows from [LP] that $X \approx$ $X \oplus l_{p}$; and from [JRZ] and [NW] that $X$ has a basis $\left(f_{n}\right)$ satisfying

$$
\sup _{n} d\left(\left[f_{i}\right]_{i=1}^{n}, l_{p}^{n}\right)=\lambda<\infty \text {. }
$$

Since

we have that

$$
l_{p} \equiv\left(\sum \bigoplus l_{p}\right)_{p}
$$

$$
X \approx \sum_{i} \bigoplus\left(f_{i} \oplus l_{p}\right)
$$

in a natural way. Now each of these summands is isomorphic to $l_{p}$; inside each we can form a continuous nest indexed by $[0,1]$ whose elements are all isomorphic to $l_{p}$. Applying the comment above and the estimate of Proposition 5.1 we obtain a continuous nest indexed by $[0, \infty]$ such that $d\left(N_{t}, l_{p}\right)<20 \lambda$ for $0<t<\infty$. Because

$$
\sum_{i=1}^{n} \bigoplus\left(f_{i} \oplus l_{p}\right) \equiv \overline{\operatorname{span}}\left(f_{i}\right)_{i=1}^{n} \oplus l_{p} \approx l_{p}
$$

with constant $\lambda$; which upon re-indexing the nest, gives the proposition. 
$(\Leftarrow)$ Let us suppose that we have a continuous nest in $X$ such that

$$
\mathbf{N}=\left\{N_{t} \mid 0 \leq t \leq 1\right\}, \quad N_{t} \approx l_{p} \quad \forall t \quad \text { and } \sup _{0 \leq t \leq 1} d\left(N_{t}, l_{p}\right)=\lambda<\infty .
$$

Let $E \subset X$ be a subspace, $\operatorname{dim} E=k$ and let $\varepsilon=1 / 3 k$ (for later use). Using the continuity of the nest at $t=1$ we can find $E^{\prime} \subset N_{t}, t<1$, such that $E^{\prime}$ is $\varepsilon$-close to $E$ (since we can do it for a single vector, we can do it for any finite number). Now $E^{\prime} \subset N_{t} \approx l_{p}$ with constant $\lambda$. Since $l_{p}$ is $\mathscr{L}_{p, 1+\delta} \forall \delta>0$, we can find a finite-dimensional subspace in $l_{p}$ which is 2close to $l_{p}$ and contains the image of $E^{\prime}$ (under the isomorphism). Pull it back into $N_{t}$, calling it $F^{\prime}$; we then have $F^{\prime} \subset N_{t}$, $\operatorname{dim} F^{\prime}=n<\infty$, with $E^{\prime} \subset F^{\prime}$ and $d\left(F^{\prime}, l_{p}^{n}\right) \leq 2 \lambda$. By a standard perturbation argument (using Lemma 2.4 of [JRZ], which requires $\varepsilon$ as above), we can obtain $F \subset X$ with $\operatorname{dim} F=\operatorname{dim} F^{\prime}, E \subset F$ and $d\left(F, l_{p}^{n}\right) \leq 4 \lambda$. Therefore $X$ is an $\mathscr{L}_{p, 4 \lambda^{-}}$ space.

The construction of a continuous nest in any space with a basis allows us to build some interesting nests. We finish with an example of a continuous nest with no two of its subspaces isomorphic.

Example. Let $X=\left(\sum_{p=3}^{\infty} \bigoplus l_{p}\right)_{2}$ with $\left(e_{n}^{p}\right)_{n=1}^{\infty}$ being the canonical basis for $l_{p}$. Construct the continuous nest as before, assigning basis elements to dyadics so as to satisfy the following:

Assign the basis elements for $l_{3}$ to dyadics in $\left[0, \frac{1}{2}\right]$.

Assign the basis elements for $l_{4}$ to dyadics in $\left[\frac{1}{2}, 1\right]$.

Assign the basis elements for $l_{5}$ to dyadics in $\left[0, \frac{1}{4}\right], \ldots$ etc. Then, given any two elements of the nest, there will be an $l_{p}$ space contained in one which is not contained in the other.

\section{REFERENCES}

[ALWW] G. D. Allen, D. R. Larson, J. D. Ward, and G. Woodward, Similarity of nests in $L_{1}$, J. Funct. Anal. 92 (1990), 49-76.

[JRZ] W. B. Johnson, H. P. Rosenthal and M. Zippin, On bases, finite-dimensional decompositions and weaker structures in Banach spaces, Israel J. Math. 9 (1971), 488-506.

[KS] R. V. Kadison and I. M. Singer, Triangular operator algebras, Amer. J. Math. 82 (1960).

[L1] D. Larson, On similarity of nests in Hilbert space and Banach spaces, Longhorn lecture notes, Springer-Verlag, New York (to appear).

[L2] _ - Triangularity in operator algebras, Surveys of Recent Results in Operator Theory (J. Conway, Ed.), Pitman Research Notes in Math., Longman (to appear).

[LP] J. Lindenstrauss and A. Pelczyński, Absolutely summing operators in $\mathscr{L}_{p}$-spaces and their applications, Studia Math. 29 (1968), 275-326.

[LT] J. Lindenstrauss and L. Tzafrifri, Classical Banach spaces. II: Function spaces, SpringerVerlag, Berlin, 1979.

[NW] N. Nielsen and P. Wojtaszczyk, A remark on bases in $\mathscr{L}_{p}$-spaces with an application of complementably universal $\mathscr{L}_{\infty}$-spaces, Bull. Acad. Sci. Polon. 21 (1973), 249-254.

[R] J. R. Ringrose, Super-diagonal forms for compact linear operators, Proc. London Math. Soc. (3) 12 (1962), 367-374.

[W] D. J. Westwood, Nests in Banach space, preprint.

Department of Mathematics, Texas A\&M University, College Station, Texas 77843

Current address, Alvaro Arias: Department of Mathematics and Statistics, Case Western Reserve University, Cleveland, Ohio 44106 